EPJ Web of Conferences 45, 01091 (2013)

DOI: $10.1051 /$ epjconf/20134501091

C Owned by the authors, published by EDP Sciences, 2013

\title{
Experimental and Numerical Research of a Novel Combustion Chamber for Small Gas Turbine Engines
}

\author{
J. Tuma ${ }^{1}$, J. Kubata $^{1}$, V. Betak ${ }^{1}$, and R. Hybl ${ }^{1}$ \\ ${ }^{1}$ Vyzkumny a zkusebni letecky ustav, a.s., 19905 Beranovych 130, Prague, Czech Republic
}

\begin{abstract}
New combustion chamber concept (based on burner JETIS-JET Induced Swirl) for small gas turbine engine (up to $200 \mathrm{~kW}$ ) is presented in this article. The combustion chamber concept is based on the flame stabilization by the generated swirl swirl generated by two opposite tangentially arranged jet tubes in the intermediate zone, this arrangement replaces air swirler, which is very complicated and expensive part in the scope of small gas turbines with annular combustion chamber. The mixing primary jets are oriented partially opposite to the main exhaust gasses flow, this enhances hot product recirculation and fuel-air mixing necessary for low NOx production and flame stability. To evaluate the designed concept a JETIS burner demonstrator (methane fuel) was manufactured and atmospheric experimental measurements of $\mathrm{CO}$, NOx for various fuel nozzles and jet tubes the configuration were done. Results of these experiments and comparison with CFD simulation are presented here. Practical application of the new chamber concept in small gas turbine liquid fuel combustor was evaluated (verified) on 3 nozzles planar combustor sector test rig at atmospheric conditions results of the experiment and numerical simulation are also presented.
\end{abstract}

\section{Introduction}

There is expected growth of air traffic in the world by $5 \%$ every year and this will reflect effect (to) higher pressure to the environment [1]. Therefore the aircraft must be optimized to be environmental friendly due to optimizations of the aircraft shape (weight and noise reduction, performance increase) and of the engine units (noise, fuel consumption and pollution reduction). Engine should be split into part of compressor/turbine and combustor.

The earlier concepts of combustion chambers were based on coaxial flow of fuel and air that had lower efficiency due to the high proportion of $\mathrm{CO}$ and NOx concentration in the exhaust gases. To reduce this phenomenon a new type of combustion chamber was designed. Inside this chamber the air and premixing fuel flows into the combustion zone from the opposite direction. There are used the air swirles for flame stabilization in big engines. This solution is very complicated and expensive for the small engines. The possibilities of using natural phenomena of flow to replace swirler have been studied in this article.

\section{Chamber configuration}

The aim was to create a stable vortex with axis aligned to the axis of fuel nozzle as is shown in figures 1 and 2 .
A position of the vortex is determined by tangentially entering primary air. The stabilization of flame in combustion chamber is achieved by high turbulence and large recirculation of hot gases back into the reaction zone which leads to mixing of combustion products with fresh mixture. It has a positive effect on the stability of combustion at high AFR in spite of low thermal NOx production by a known Zeldovic mechanism.

The experimental chamber was constructed as a planar segment with three nozzles. This construction allows measurements with PIV and OH-PLIF. Only central nozzle was measured by the PIV and OH-PLIF, two lateral nozzles are used (present) for simulation of cyclic boundary conditions only (full annular combustor have 12 fuel nozzles). The actual optical input of laser plane into the primary zone area is ensured through the window from synthetic fused $\mathrm{SiO}_{2}$ located beside central nozzle under defined angle. Side windows ensure visibility of primary zone. For preliminary tests with tubular model of combustion chamber with one nozzle methane was used for its relatively well-known and the simplest chemical and physical properties. There was used JET A1 as a test fuel with segment of combustion chamber (3 nozzles) in experiments.

Compared to the traditional small combustion chambers (mostly RQL concept) construction of JETIS has a potential to produce less $\mathrm{CO}$ emissions and also ensure better durability of combustor due to separation of hot zone from walls by swirl. 


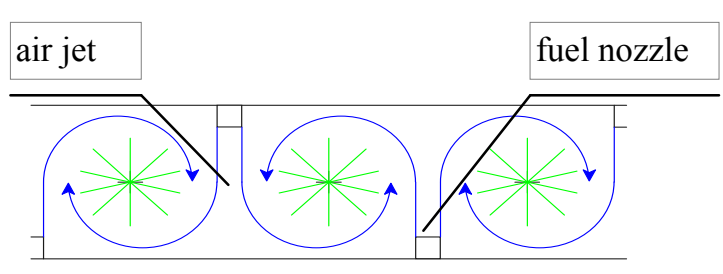

Fig. 1. Vortex position

There is shown in figure 2 the tested construction of chamber with schemes of vortices. Main swirl (yellow line) is ensured by jets of fresh air (blue lines) and fuel from nozzles entering to the chamber in direction of green axis.

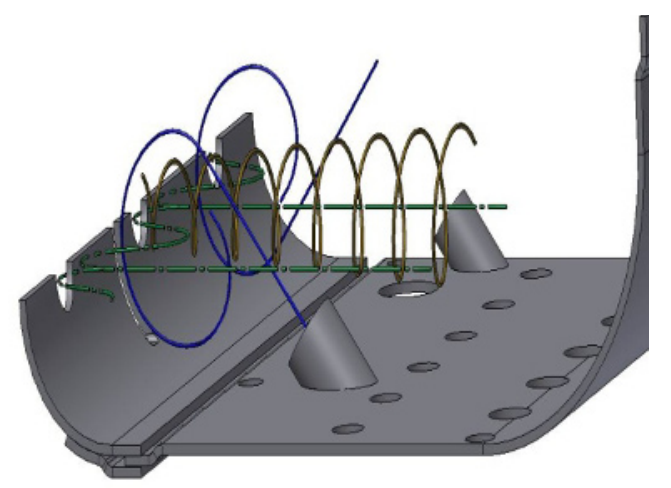

Fig. 2. Schemes of vortex in planar combustion chamber

\section{Test facilities}

Two test facilities were built. The first is JETIS demonstrator - burner (figure 3) and the test facility for linear segment of combustion chamber (figure 4) with optical access for PIV and OH-PLIF measurements. JETIS-burner facility was designed for a preliminary test to verify this concept of a combustion chamber especially the stability of combustion. JETIS-burner is an ideal model of combustion chamber segment with only one nozzle. Second one is designed to verify stability, emissions and temperature field in conditions close to real full annular combustion chamber. Both facilities were designed to respect similarity of inlet temperature and flow number. Required thermodynamic parameters are controlled by adjustment of source engine power, which was used as source of heated and compressed air. Elevated pressure conditions were achieved by a valve at the exit of the chamber. Experimentally obtained data were used for calibration of numerical models.

\section{Equations and mathematics}

Lots of numerical methods have been tested; where the efficiency based on ratio between time consumption and accuracy of calculation have been compared. The model based on unsteady Reynolds Averaged Navier Stokes
(RANS) equations have been chosen for the best efficiency that was used in following form

$$
\begin{gathered}
\frac{\partial \rho}{\partial t}+\frac{\partial}{\partial x_{j}}\left(\rho u_{j}\right)=0 \\
\frac{\partial}{\partial t}\left(\rho u_{i}\right)+\frac{\partial}{\partial x_{j}}\left(\rho u_{i} u_{j}\right)=-\frac{\partial p}{\partial x_{i}}+ \\
+\frac{\partial}{\partial x_{j}}\left[\left(\mu+\mu_{t}\right)\left(\frac{\partial u_{i}}{\partial x_{j}}+\frac{\partial u_{j}}{\partial x_{i}}-\frac{2}{3} \frac{\partial u_{k}}{\partial x_{k}} \delta_{i j}\right)\right]+S_{D}^{u} \\
\frac{\partial h}{\partial t}+\frac{\partial}{\partial x_{j}}\left(h u_{j}\right)=\frac{D p}{D t}+\frac{\partial}{\partial x_{j}}\left[\left(\alpha+\frac{\mu_{t}}{P r_{t}}\right) \frac{\partial h}{\partial x_{j}}\right]+ \\
+S_{D}^{u}+S_{C h}^{b}
\end{gathered}
$$

where $\rho$ mean density, $u$ velocity, $p$ pressure and $h$ enthalpy. We need to know a turbulent dynamic viscosity $\mu$, thermal diffusivity $\alpha$ and Prandtl turbulent number Pr for the simulation. The sources $\mathrm{S}_{\mathrm{d}}^{\mathrm{u}}$ described the influence of droplets to the flow filed, $\mathrm{S}_{\mathrm{d}}^{\mathrm{k}}$ is evaporated heat and $\mathrm{S}_{\mathrm{Ch}}^{\mathrm{b}}$ is combustion heat. The coupling of pressure and velocity is done by PISO algorithm.

The assumption of log law validity has been applied to reduce hardware requirements of simulations. There was

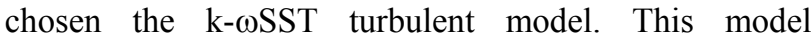
significantly reduces the temperature overshoot of $\mathrm{k}-\varepsilon$ model with similar computational efficiency. This model is described by following system of equations

$$
\begin{gathered}
\frac{\partial}{\partial t}(\rho k)+\frac{\partial}{\partial x_{j}}\left(\rho k u_{j}\right)=\frac{\partial}{\partial x_{j}}\left[\Gamma_{k} \frac{\partial k}{\partial x_{j}}\right]+G_{k}-Y_{k}, \\
\frac{\partial}{\partial t}(\rho \omega)+\frac{\partial}{\partial x_{j}}\left(\rho \omega u_{j}\right)=\frac{\partial}{\partial x_{j}}\left[\Gamma_{\omega} \frac{\partial \omega}{\partial x_{j}}\right]+G_{\omega}-Y_{\omega}+D_{\omega}
\end{gathered}
$$

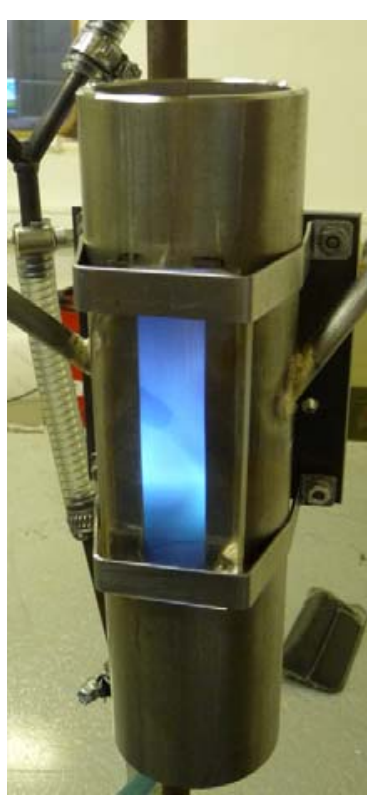

Fig. 3. JETIS burner 


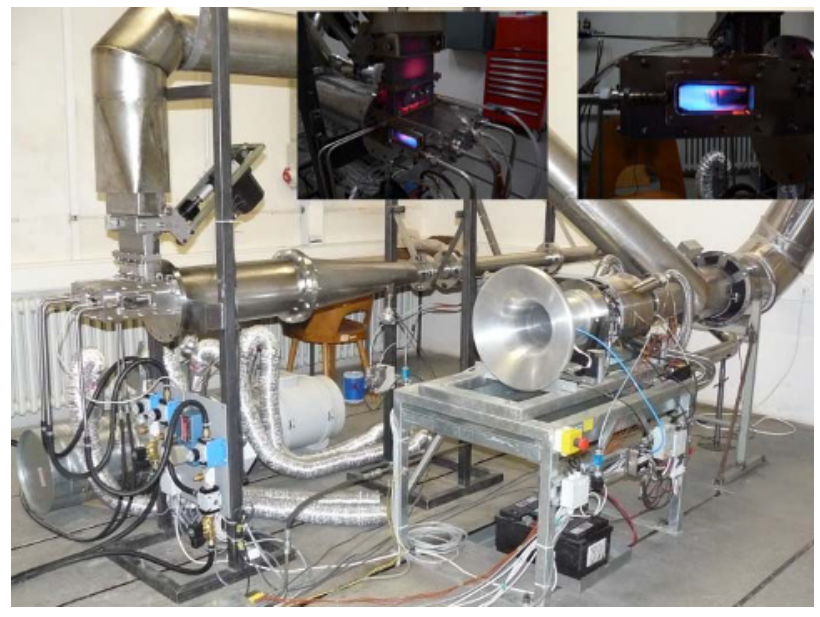

Fig. 4. Test facility for linear segment

and

$$
\mu_{t}=\frac{\rho k}{\omega} \frac{1}{\max \left(\frac{1}{\alpha}, \frac{S F_{2}}{a_{1} \omega}\right)}
$$

where $\mathrm{k}$ is turbulent kinetic energy and $\omega$ is a specific dissipation rate. $G_{k}, G_{\omega}, Y_{k}, Y_{\omega}, D_{\omega}$ are model functions that are define e.g. [3]

Additional transport equations for components of the mixture have to be solved. This is shown in the next PDE equation

$$
\frac{\partial}{\partial t}\left(\rho Y_{i}\right)+\frac{\partial}{\partial x_{j}}\left(\rho Y_{i} u_{j}\right)=\frac{\partial}{\partial x_{j}}\left[\left(\mu+\mu_{t}\right) \frac{\partial Y_{i}}{\partial x_{j}}\right]+S_{D}+S_{C h}
$$

where $Y_{t}$ is a component of mixture, $S_{d}$ is evaporated mass and $\mathrm{S}_{\mathrm{Ch}}$ is a burned mass.

If combustion of liquid fuel is solved then the movement of particles which is described by the following ODE has to be taken into account

$$
\begin{gathered}
\frac{d u_{p}}{d t}=F_{D}\left(u-u_{p}\right)+g \frac{\rho_{p}-\rho}{\rho_{p}}+F_{x}, \\
F_{D}=\frac{18}{24} \frac{\rho}{\rho_{p} d_{p}} C_{D} \% R e, \\
F_{X}=\frac{\rho}{\rho_{p}}\left[0.5 \frac{d}{d t}\left(u-u_{p}\right)+u_{p} \frac{\partial u}{\partial x}\right], \\
R_{e}=\rho d_{p} \frac{\left(u-u_{p}\right)}{\mu} \text { and } C_{D}=\frac{24}{R_{e}}
\end{gathered}
$$

where $u_{p}$ is a speed of particle, $\rho_{p}$ is a density of fuel and $d_{p}$ is a diameter of particle.
The solution of chemistry is based on non-premixed model. The chemical kinetics of it was described by the reduced mechanism. The stability of simulations with these mechanisms was higher and allows to describe the burning of multicomponent liquid fuel in easy way. For case of methane burner were used different types of reduced mechanisms with different number of steps. The examples of these models for methane chemistry are shown in following tables

$$
k=A\left(\frac{T}{T_{0}}\right)^{B} e^{\frac{-E_{a}}{R T}}
$$

Table 1. One-step reduced chemistry of methane

\begin{tabular}{|c|l|l|l|l|}
\hline step & equations & A & B & $\mathbf{E}_{\mathbf{a}}$ \\
\hline $\mathrm{I}$ & {$\left[\mathrm{CH}_{4}\right]^{0.2}+\left[2 \mathrm{O}_{2}\right]^{1.3} \Rightarrow \mathrm{CO}_{2}+2 \mathrm{H}_{2} \mathrm{O}$} & $2.11 \mathrm{E}+12$ & 0.00 & 38414.06 \\
\hline
\end{tabular}

Table 2. Four-step reduced chemistry of methane [3]

\begin{tabular}{|c|l|l|l|l|}
\hline step & equations & A & B & $\mathbf{E}_{\mathbf{a}}$ \\
\hline I & {$\left[\mathrm{CH}_{4}\right]^{0.5}+\left[0.5 \mathrm{O}_{2}\right]^{1.25}=>\mathrm{CO}+2 \mathrm{H}_{2}$} & $7.82 \mathrm{E}+13$ & 0.00 & 30000 \\
\hline II & {$\left[\mathrm{CH}_{4}\right]^{1}+\left[\mathrm{H}_{2} \mathrm{O}\right]^{1}=>\mathrm{CO}+3 \mathrm{H}_{2}$} & $3 \mathrm{E}+11$ & 0.00 & 30000 \\
\hline III & {$\left[\mathrm{H}_{2}\right]^{0.5}+\left[0.5 \mathrm{O}_{2}\right]^{1.5}<=>\left[\mathrm{H}_{2} \mathrm{O}\right]^{1}$} & $1.21 \mathrm{E}+18$ & 0.00 & 40000 \\
\hline IV & {$[\mathrm{CO}]^{1}+\left[\mathrm{H}_{2} \mathrm{O}\right]^{1}<=>\left[\mathrm{CO}_{2}\right]^{1}+\left[\mathrm{H}_{2}\right]^{1}$} & $2.75 \mathrm{E}+12$ & 0.00 & 20000 \\
\hline
\end{tabular}

where A, B and Ea are the coefficient in Arhenius equations.

The alternative chemistry and its reduced mechanism for liquid fuel Jet-A1 is based on the principle of replacement of multicomponent compounds (eg. fuel Jet A1) by one-component compound with the same physical and chemical properties. The new compound was derived by mass averaging from original compound with following formula $\mathrm{C}_{12} \mathrm{H}_{23}$. The proximity of some physical properties to $\mathrm{C}_{12} \mathrm{H}_{26}$ were assumed. Other properties such as density, vapor pressure, surface tension and heat capacity were derived from data provided by producer. The following three steps reaction model was applied.

Table 3. Three steps reduced chemistry of Jet A1

\begin{tabular}{|c|l|l|l|l|}
\hline step & equations & A & B & Ea \\
\hline I & {$\left[\mathrm{C}_{12} \mathrm{H}_{23}\right]^{1}+\left[11.75 \mathrm{O}_{2}\right]^{0.5} \begin{array}{r}12 \mathrm{CO}+ \\
11.5 \mathrm{H}_{2} \mathrm{O}\end{array}$} & $1.04 \mathrm{E}+9$ & 0.00 & 20073.0 \\
\hline II & {$[\mathrm{CO}]^{1}+\left[0.5 \mathrm{O}_{2}\right]^{0.5}=>\mathrm{CO}_{2}$} & $4.04 \mathrm{E}+8$ & 0.00 & 12008.0 \\
\hline III & {$\left[\mathrm{N}_{2}\right]^{1}+\left[\mathrm{O}_{2}\right]^{0.5}=>2 \mathrm{NO}$} & $7.4 \mathrm{E}+13$ & -0.5 & 76337.13 \\
\hline
\end{tabular}

\section{Results}

The study of JETIS burner was done in the first part of this project. The relationship between emissions and Air Fuel Ration (AFR) is presented in Fig. 5. Based on this analysis the optimal AFR in primary zone is around 35 . 


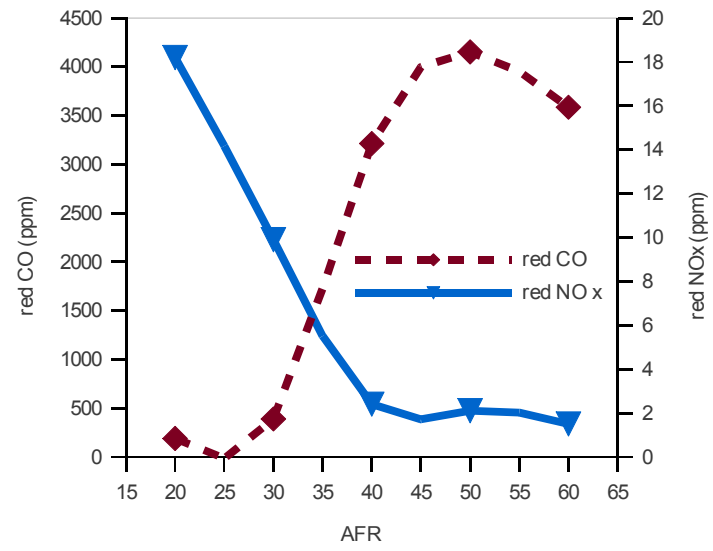

Fig. 5. Reduced emissions for different Air Fuel Ratio

$$
A F R=\frac{m_{\text {air }}}{m_{\text {fuel }}}[-]
$$

Figure 6. shown an example of numerical simulations where a magnitude of turbulent viscosity and flame (rate of chemistry reaction) position is visible. The inlet of fresh air is in position of lower bottle-neck on left picture. Turbulence around primary zone ensures the stability of combustion.

Following results are obtained from preliminary planar demonstrator of combustion chamber. High speed camera was tested as additional visualization equipment for its simple use (figure 8).
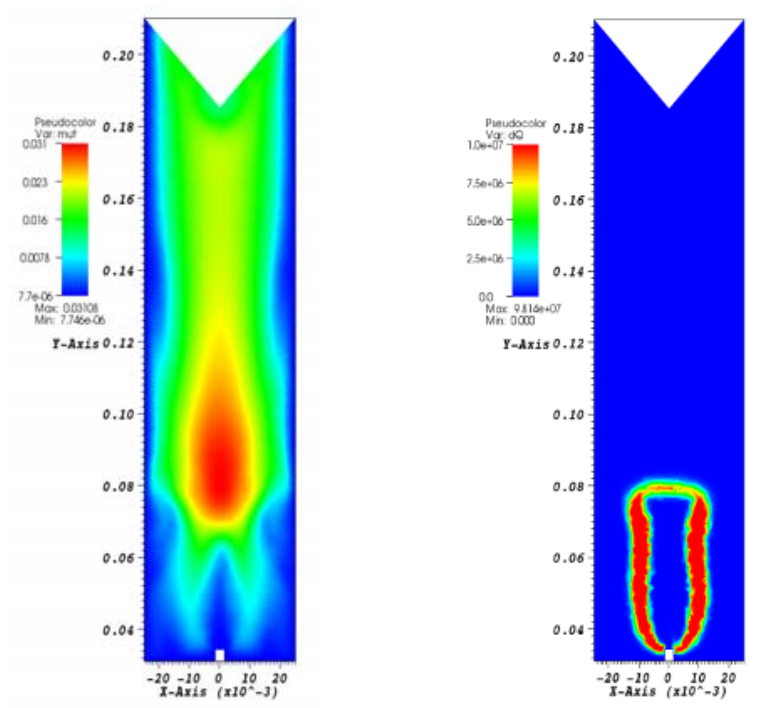

Fig. 6. Magnitude of turbulent viscosity (left) and flame position (right)
There were used OH-PLIF measurements [4] to compare the results of CFD simulation and verification of their accuracy. Measurements of OH radicals field (OH PLIF) were allowed by the side windows from fused $\mathrm{SiO}_{2}$. Dimensions of the front entry and laser side windows were selected to ensure maximum visibility of the primary zone. Inspected zone is shown in figure. 4. In figure 9 and figure 10 there is visualization of primary zone. Time averaged data measured by OH-PLIF (and figure 9) is possible to compare with results from CFD simulations. Triangular shape on the bottom of and figure 8 is a tube with primary air jet, which shields the CCD camera. In CFD results (and figure 10) this tube is not visible. Angle of laser sheet input into the combustion chamber segment and CFD cut is same.

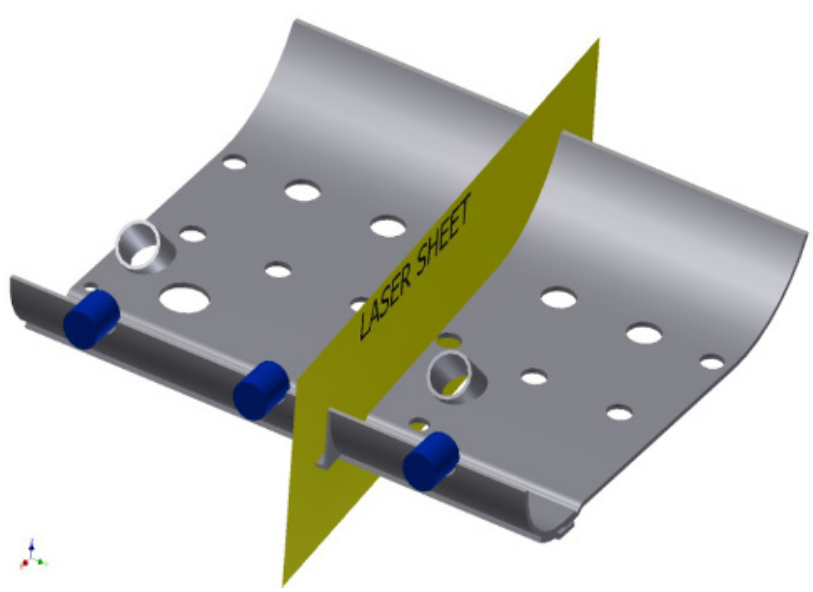

Fig. 7. Position of laser sheet in combustion chamber segment

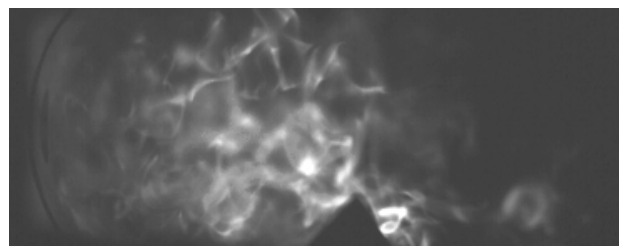

Fig. 8. High speed camera figure of flame's [4]

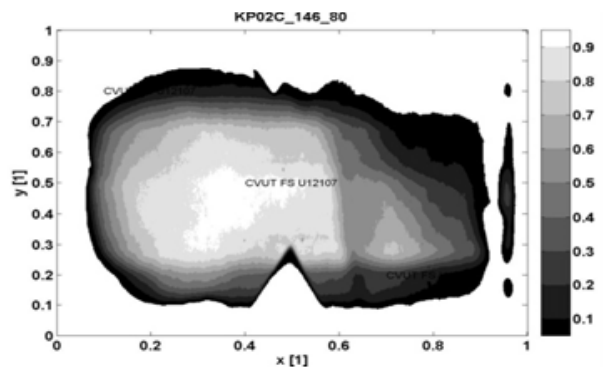

Fig. 9. PLIF results of flame position in planar combustion chamber [4] 


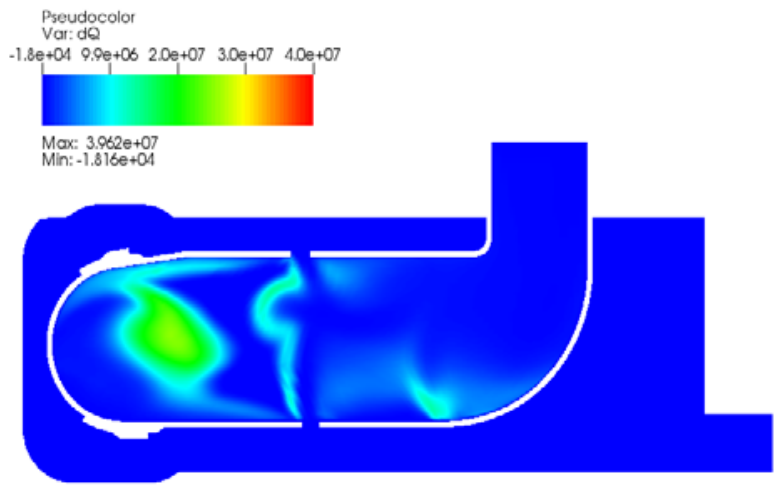

Fig. 9. Numerical results of flame position in planar combustion chamber

\title{
4 Conclusion
}

Idea of new type of combustion chamber was developed based on principle of JET Induced Swirl. This concept significantly reduces $\mathrm{CO}$ and $\mathrm{NOx}$ emissions in comparison to the traditional RQL concepts. This new type of chamber also eliminates pressure fluctuation and instability in burning process. A future work will be oriented on improvement of the performance of combustion chamber and optimization of CFD tools.

\section{Nomenclature}

\author{
JETIS $=$ JET Induced Swirl \\ OH-PLIF = Planar Laser-Induced Fluorescence \\ imaging of $\mathrm{OH}$ radicals \\ $\mathrm{PIV}=$ Particle Image Velocimetry \\ RQL = Rich burn-Quick Quench-Lean burn \\ RANS $=$ Reynolds Avereged Navier Stokes
}

\section{References}

1. A. Mastorakos, Cambridge university-lecture proceedings

2. CH. Westbrook, F.L. Dryer, Prog. Energy sci., 10, 157(1984)

3. B.F.W. Gschaider, M. Rehm, P. Seifert, B. Meyer, Open Source CFD International Conference 2008, (2008)

4. J. Matecha, J. Novotny, Research of new concept of combustion chamber C(P)DT, CTU Prague, (2009) 